www.jestr.org

\title{
Model of Gross Error Elimination for Monitoring Data Collected by A Surveying Robot
}

\author{
Yachun Mao $^{1, *}$, Yongsheng Chen ${ }^{1}$, Shuhong Wang ${ }^{1}$ and Roaj Betoubert ${ }^{2}$ \\ ${ }^{1}$ School of Resources \& Civil Engineering, Northeastern University, Shenyang, 110819, China \\ ${ }^{2}$ Universidad Andres Bello, Avenida República 220, 837-0134 Santiago, Chile
}

Received 19 May 2014; Accepted 15 September 2014

\begin{abstract}
The 3D monitoring system based on a surveying robot and the Geomos software is widely used in the slope monitoring of open pits. The effect of various factors, such as refraction and blasting vibration, is crucial to the monitoring process. The probability of monitoring data to contain gross error is significantly higher than $0.3 \%$. Thus, using the Paūta criterion as the elimination criterion of the gross error is unreasonable. In this study, the data on multi-period monitoring were used as the source, and the accuracy requirements of the Code for Engineering Survey (2007) were set as the goal of the slope monitoring of open pits. The actual probability of the monitoring data to contain gross error was calculated through mathematical statistics. An elimination model of gross error was developed based on probability theory. The practicality of the proposed model was tested vis-à-vis the practical data on the slope monitoring of open pits.
\end{abstract}

Keywords: Slope monitoring, Surveying robot, Gross error, Paūta criterion

\section{Introduction}

After a long period of mining, the depth and slope length of an open pit increase, and its slope stability deteriorates progressively. The recent local landslides at the open-pit iron mine of Qidashan in Anshan, China are shown in Figure 1.

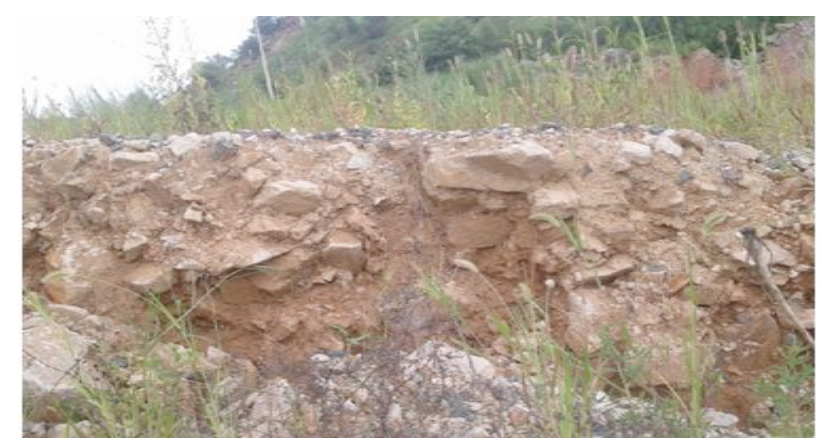

a) The first landslide area

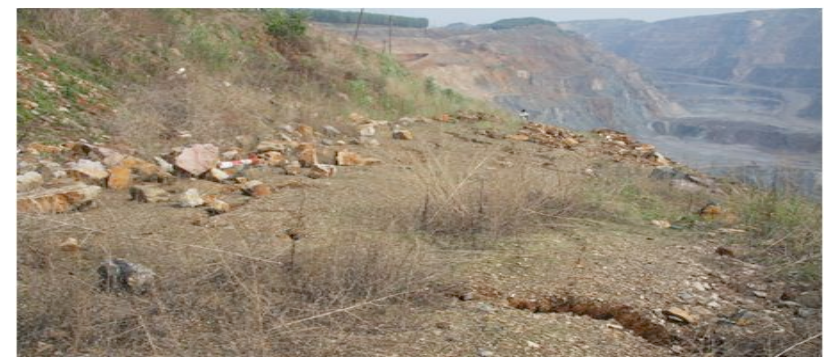

b) The second landslide area

Fig. 1. Local slope displacements at the open-pit iron mine in Qidashan, Anshan
Many scholars have conducted in-depth research on early warning systems for landslides [1, 2], monitoring methods, processing methods of monitoring data, and slope stability analysis [3-8] to solve the warning problems related to the slope safety of open pits. The $3 \mathrm{D}$ monitoring and warning system based on a surveying robot has been widely used in slope monitoring [9-13]. Figure 2 shows the monitoring sites of the $3 \mathrm{D}$ monitoring system based on a surveying robot at the open-pit iron mines in Qidashan and Dagushan. Nevertheless, the methods of gross error elimination based on monitoring data collected by a surveying robot have not been studied extensively. Thus, real-time early warning systems and rapid processing of monitoring data remain limited.

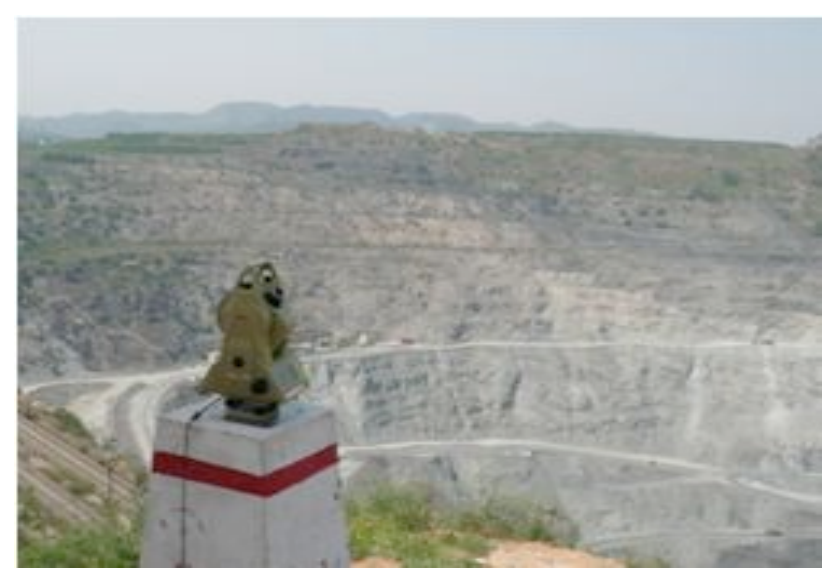

a) The Dagushan open-pit iron mine

*E-mail address: DBDXMYC@163.com

ISSN: $1791-2377$ (C) 2014 Kavala Institute of Technology. All rights reserved. 


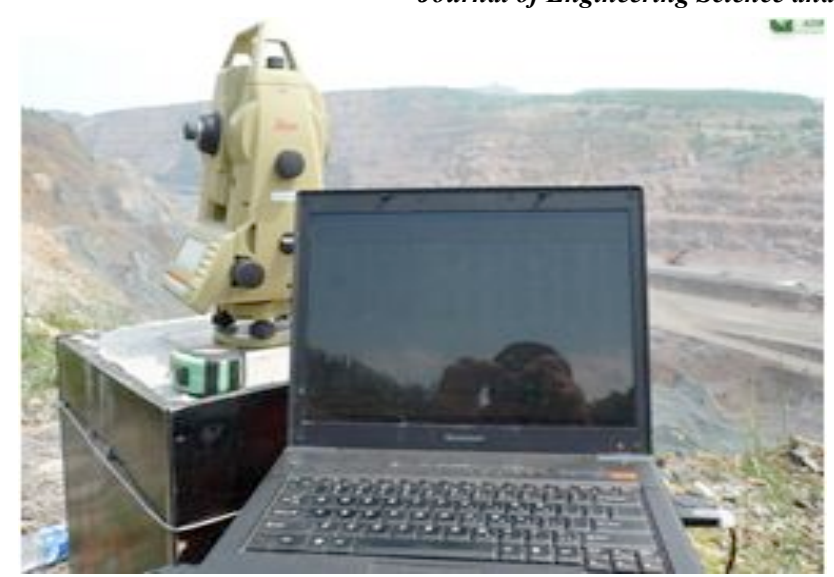

b) The Qidashan open-pit iron mine

Fig. 2. Monitoring site at (a) Dagushan and (b) Qidashan open-pit iron mines in Anshan

The rationality and practicability of using the Paūta criterion as the elimination model of the gross error data collected by a surveying robot are worth studying further. The Paūta criterion is often used as theoretical basis for the gross error elimination of monitoring data in various engineering projects[14-17]. When $v_{\mathrm{i}} \geq 3 \delta$, where $v_{\mathrm{i}}$ is the residual of observations $\left(L_{\mathrm{i}}\right)$ and $\delta$ is the standard deviation, $L_{\mathrm{i}}$ is referred to as the observations that contain the gross error when the error probability is assumed to be $0.3 \%$.

However, the probabilities of the gross error of various monitoring data differ because of the varying stability of different monitoring equipment and environments. The assumption that the probability of gross error is $0.3 \%$ is unscientific. Studies have found that the probability of monitoring data to contain gross error is significantly higher than $0.3 \%$, particularly when the $3 \mathrm{D}$ monitoring system based on a surveying robot and Geomos software is used under the worst climate conditions, such as severe atmospheric refraction and blasting vibrations. Using $v_{\mathrm{i}} \geq 3 \delta$ as the elimination model of the gross error often leads to failure. The processed results do not meet the accuracy requirements of open-pit slope monitoring, which has become the bottleneck of real-time warning.

In this study, the precision of azimuths and vertical angles is analyzed using the law of error propagation. A total of 24 cycles of monitoring data on the Qidashan, Yanqianshan, and Dagushan open-pit iron mines are used as data source. According to the accuracy requirements of the rock landslide monitoring in the Code for Engineering Survey (2007), the precision of azimuths is used as the indicator of eliminating gross error. The actual probability of observations containing gross error has been analyzed through mathematical statistics. The model of gross error elimination based on monitoring data collected by a surveying robot was developed. The practicality of the proposed model was tested vis-à-vis the practical data of slope monitoring.

\section{Precision Analysis of Monitored Points}

The basic data collected by a surveying robot includes horizontal angle, vertical angle, and slope distance. Figure 3 shows that $k_{1}$ and $k_{2}$ are the station and back sight points, and their coordinates are $x_{k 1}, y_{k 1}, x_{k 2}$, and $y_{k 2} . \alpha_{K 1-K 2}$ is the azimuth between station point $k_{1}$ and back sight point $k_{2}$. Given their orientation deviation, the actual measured azimuths $\alpha_{K 1-K 2}^{\prime}$ and $\alpha^{\prime}$ are not equal to $\alpha_{K 1-K 2}$ and $\alpha$. This difference has to be corrected when calculating the direction. $S$ is the slope distance between station point $k_{1}$ and monitoring point $A$.

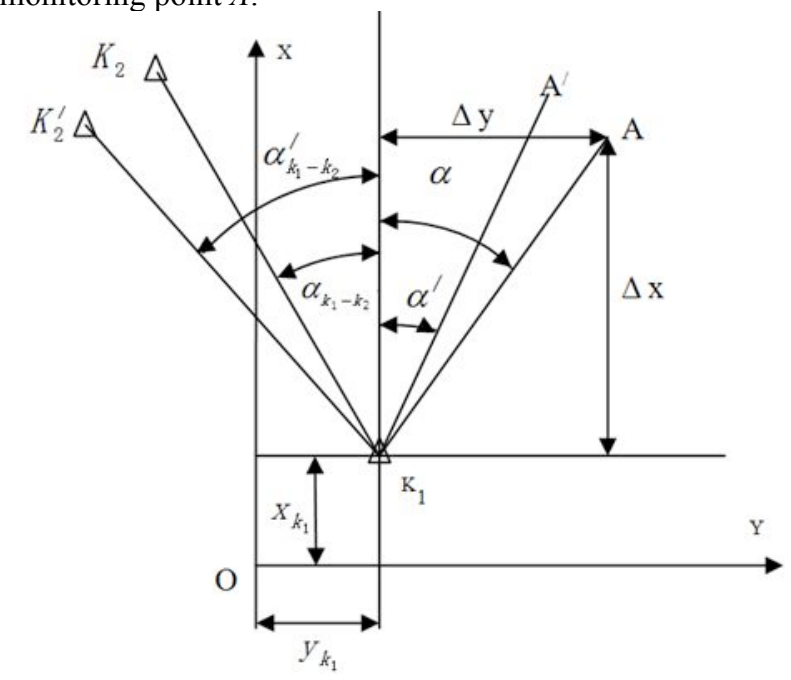

Fig. 3. Monitoring point location

If the vertical angle between station point $k_{1}$ and monitoring point $A$ is $\beta$, then the increment in the coordinates of station point $k_{1}$ and monitoring point $A$ are $\Delta x, \Delta y$. The coordinates of monitoring point $A$ are calculated as follows:

$$
\begin{aligned}
& \mathrm{x}_{\mathrm{A}}=\mathrm{x}_{\mathrm{k}_{1}}+\Delta \mathrm{x}=\mathrm{x}_{\mathrm{k}_{1}}+\mathrm{s} \times \cos \beta \times \cos \alpha \\
& \mathrm{y}_{\mathrm{A}}=\mathrm{y}_{\mathrm{k}_{1}}+\Delta \mathrm{y}=\mathrm{y}_{\mathrm{k}_{1}}+\mathrm{s} \times \cos \beta \times \sin \alpha \\
& \alpha=\alpha^{\prime}+\alpha_{\mathrm{K}_{1}-\mathrm{K}_{2}}-\alpha_{\mathrm{K}_{1}-\mathrm{K}_{2}}^{\prime}
\end{aligned}
$$

Using the error propagation, we obtain:

$$
\begin{aligned}
& m_{x_{A}}^{2}=m_{x_{k 1}}^{2}+m_{\Delta_{x}}^{2} \\
& m_{y_{A}}^{2}=m_{y_{k 1}}^{2}+m_{\Delta_{y}}^{2} \\
& m_{\alpha}^{2}=m_{\alpha^{\prime}}^{2}+m_{\alpha_{k_{1}-k_{2}}}^{2}+m_{\alpha_{k_{1}-k_{2}}^{\prime}}^{2}
\end{aligned}
$$

$m_{\Delta \mathrm{x}}^{2}=\left(\frac{\partial_{\Delta \mathrm{x}}}{\partial_{s}}\right)^{2} \times m_{s}^{2} \times \cos ^{2} \beta \times \cos ^{2} \alpha+\left(\frac{\partial_{\Delta \mathrm{x}}}{\partial_{\beta}}\right)^{2} \times \frac{m_{\beta}^{2}}{\rho^{2}} \times s^{2} \times \cos ^{2} \alpha+\left(\frac{\partial_{\Delta \mathrm{x}}}{\partial_{\alpha}}\right)^{2} \times \frac{m_{\alpha}^{2}}{\rho^{2}} \times s^{2} \times \cos ^{2} \beta$

$m_{\Delta \mathrm{x}}^{2}=m_{s}^{2} \times \cos ^{2} \beta \times \cos ^{2} \alpha+\frac{m_{\beta}^{2}}{\rho^{2}} \times \sin ^{2} \beta \times s^{2} \times \cos ^{2} \alpha+\frac{m_{\alpha}^{2}}{\rho^{2}} \times \sin ^{2} \alpha \times s^{2} \times \cos ^{2} \beta$ 
Yachun Mao, Yongsheng Chen, Shuhong Wang and Roaj Betoubert /

Journal of Engineering Science and Technology Review 7 (4) (2014) 13 - 17

$m_{\Delta y}^{2}=\left(\frac{\partial_{\Delta y}}{\partial_{s}}\right)^{2} \times m_{s}^{2} \times \cos ^{2} \beta \times \sin ^{2} \alpha+\left(\frac{\partial_{\Delta y}}{\partial_{\beta}}\right)^{2} \times \frac{m_{\beta}^{2}}{\rho^{2}} \times s^{2} \times \sin ^{2} \alpha+\left(\frac{\partial_{\Delta y}}{\partial_{\alpha}}\right)^{2} \times \frac{m_{\alpha}^{2}}{\rho^{2}} \times s^{2} \times \cos ^{2} \beta$

$m_{\Delta y}^{2}=m_{s}^{2} \times \cos ^{2} \beta \times \sin ^{2} \alpha+\frac{m_{\beta}^{2}}{\rho^{2}} \times \sin ^{2} \beta \times s^{2} \times \sin ^{2} \alpha+\frac{m_{\alpha}^{2}}{\rho^{2}} \times \cos ^{2} \alpha \times s^{2} \times \cos ^{2} \beta$

where $\rho=206265$ (seconds of one radian). Because $k_{1}$ and $k_{2}$ are the controlling points, the station is set up by forced centering, which means that $m_{x_{k 1}}^{2}, m_{y_{k 1}}^{2}$, and $m_{\alpha_{k 1}{ }^{-}{ }_{2}}^{2}$ may be zero. $\alpha^{\prime}$ and $\alpha_{K 1-K 2}^{\prime}$ are measured under the same observation conditions, $m_{\alpha^{\prime}}^{2}=m_{\alpha_{k 1-k 2}}^{2}$;therefore, $m_{\alpha}^{2}=2 m_{\alpha^{\prime}}^{2}$. The mean square error (MSE) of the monitoring point can be expressed as follow:

$$
m_{L}^{2}=m_{\Delta x}^{2}+\mathrm{m}_{\Delta y}^{2}=\mathrm{m}_{s}^{2} \times \cos ^{2} \beta+\frac{s^{2} \times m_{\beta}^{2}}{\rho^{2}} \times \sin ^{2} \beta+\frac{s^{2} \times 2 m_{\alpha^{\prime}}^{2}}{\rho^{2}} \times \cos ^{2} \beta
$$

According to the adjustment of multiple-period actual monitoring data, the precision of the vertical angle and azimuth are almost the same. Therefore,

$\mathrm{m}_{\alpha^{\prime}} \approx \mathrm{m}_{\beta}=\mathrm{m}$

Substituting Equation (10) into Equation (9), we obtain:

$m_{L}^{2}=m_{s}^{2} \times \cos ^{2} \beta+\frac{s^{2} \times m^{2}}{\rho^{2}}+\frac{s^{2} \times m^{2}}{\rho^{2}} \times \cos ^{2} \beta$

According to the requirements of the Code for Engineering Survey (2007), the MSE of rock slope monitoring points should be $\pm 6 \mathrm{~mm}$, which means that:

$\left|m_{L}\right|=\sqrt{m_{\Delta x}^{2}+m_{\Delta y}^{2}} \leq 6 m m$

Based on the processing results of 24 cycles of monitoring data at three open-pit mines, the precision of the 10 to 15 measured values can generally be as precise as a submillimeter, so $m_{s}^{2}$ has limited effect on the MSE of the monitoring points. Equation (11) can then be expressed as follow:
$m_{L}^{2}=\frac{s^{2} \times m^{2}}{\rho^{2}}+\frac{s^{2} \times m^{2}}{\rho^{2}} \times \cos ^{2} \beta$

$m_{L}$ reaches its maximum when $\beta$ is zero.

To ensure the practicality of the developed model, we let $\beta=0$. Therefore, Equation (13) becomes:

$m_{L}=\frac{\sqrt{2} m}{\rho} \times s$

The surveying robot can automatically be monitored under the control of Geomos software, but the ATR function of the surveying robot (TCA2003) must be enabled. The maximum measuring range is $1 \mathrm{~km}$ so that the longest measuring distance is $1 \mathrm{~km}$ of the slope distance, that is, $\mathrm{S}=$ $1000 \mathrm{~m}$, which when substituted into Equation (14) yields:

$\frac{\sqrt{2} m}{\rho} \times 10^{3} \leq 0.006$

$|m| \leq \frac{0.006}{\sqrt{2} \times 10^{3}} \times \rho=0.8^{\prime \prime}$

To ensure the practicality of the developed model for multiple observation monitoring data, the MSE of one observation should be smaller than or equal to $0.8^{\prime \prime} \sqrt{n}$, where $\mathrm{n}$ is the number of observation data.

\section{Analysis of Probability of Actual Gross Error}

The probabilities of the azimuth and vertical angles containing gross errors in 24 cycles of the monitoring data were calculated, as shown in Tables 1 and 2, respectively.

Table 1. Probabilities of azimuth containing gross errors

\begin{tabular}{|c|c|c|c|c|c|c|c|c|}
\hline Cycle & 1 & 2 & 3 & 4 & 5 & 6 & 7 & 8 \\
\hline Probability & $3.2 \%$ & $3.6 \%$ & $4.1 \%$ & $2.9 \%$ & $3.3 \%$ & $4.2 \%$ & $2.9 \%$ & $2.8 \%$ \\
\hline Cycle & 9 & 10 & 11 & 12 & 13 & 14 & 15 & 16 \\
\hline Probability & $3.1 \%$ & $3.4 \%$ & $3.2 \%$ & $3.8 \%$ & $3.7 \%$ & $3.5 \%$ & $3.6 \%$ & $3.4 \%$ \\
\hline Cycle & 17 & 18 & 19 & 20 & 21 & 22 & 23 & 24 \\
\hline Probability & $3.0 \%$ & $2.9 \%$ & $3.3 \%$ & $3.6 \%$ & $3.4 \%$ & $2.7 \%$ & $3.3 \%$ & $3.5 \%$ \\
\hline
\end{tabular}

Table 2. Probabilities of vertical angle containing gross errors

\begin{tabular}{|c|c|c|c|c|c|c|c|c|}
\hline Cycle & 1 & 2 & 3 & 4 & 5 & 6 & 7 & 8 \\
\hline Probability & $3.7 \%$ & $2.9 \%$ & $3.8 \%$ & $2.6 \%$ & $3.4 \%$ & $4.0 \%$ & $3.1 \%$ & $3.2 \%$ \\
\hline Cycle & 9 & 10 & 11 & 12 & 13 & 14 & 15 & 16 \\
\hline Probability & $3.3 \%$ & $3.5 \%$ & $3.6 \%$ & $3.5 \%$ & $3.4 \%$ & $3.4 \%$ & $3.2 \%$ & $3.6 \%$ \\
\hline Cycle & 17 & 18 & 19 & 20 & 21 & 22 & 23 & 24 \\
\hline Probability & $3.2 \%$ & $3.3 \%$ & $3.0 \%$ & $3.3 \%$ & $3.5 \%$ & $3.1 \%$ & $3.5 \%$ & $3.6 \%$ \\
\hline
\end{tabular}

The average of the probability of the azimuth containing gross errors is $3.4 \%$ and the maximum is $4.2 \%$.
The average of the probability of the vertical angle containing gross errors is $3.4 \%$ and the maximum is $4.0 \%$. 
Yachun Mao, Yongsheng Chen, Shuhong Wang and Roaj Betoubert /

Journal of Engineering Science and Technology Review 7 (4) (2014) 13 - 17

To ensure the practicality of the developed model, we use $4.2 \%$ as the probability of occurrence of gross errors.

\section{Model of Gross Error Elimination}

The errors of the monitoring data measured by the surveying robot include systemic and random errors. Systemic errors can be removed through dual side observation. The remaining errors are basically random errors that follow the normal distribution. According to probability and mathematical statistics, when $\xi \sim \mathrm{N}(\mu, \delta 2), \frac{\xi-\mu}{\sigma} \sim \mathrm{N}(0,12)$, we obtain:

$$
P(a<\xi<b)=\Phi\left(\frac{b-\mu}{\sigma}\right)-\Phi\left(\frac{a-\mu}{\sigma}\right)
$$

where $\Phi(x)=\frac{1}{\sqrt{2 \pi}} \int_{-\infty}^{x} e^{-\frac{t^{2}}{2}} d t=\frac{1}{2}+\frac{1}{\sqrt{2 \pi}} \int_{0}^{x} e^{-\frac{t^{2}}{2}} d t$

If we let $H(x)=\int_{-\infty}^{x} e^{-\frac{t^{2}}{2}} d t$, then the following equations can be obtained: $e^{t} \approx 1+t+\frac{t^{2}}{2 !}+\frac{t^{3}}{3 !}+\cdots+\frac{t^{10}}{10 !}$.

According to precision requirement in this study, the first six items are taken, so we obtain:

$$
\begin{aligned}
& \frac{2}{\sqrt{2 \pi}} \int_{0}^{x} e^{-\frac{t^{2}}{2}} d t=\frac{2}{\sqrt{2 \pi}} H(x)=(1-0.042)=0.958 \\
& \frac{\sqrt{2 \pi}}{2} 0.958=H(x)
\end{aligned}
$$

Solving Equation (18), we obtain $\mathrm{x}=2.1$ so that the model of gross error elimination for the azimuth and vertical angle measured by the surveying robot may be described as follow:

$$
v_{i} \geq 2.1 \delta
$$

Thus, when the residuals of the azimuth and vertical angle $v_{i} \geq 2.1 \delta$, they are considered as the observations containing gross errors.

\section{Practical Test of Developed Model}

Table 3 shows the values of the azimuth and residual of point 2 at the Qidashan iron mine in Anshan on August 10 2012. The average of the azimuths is $171.8712970^{\circ}$, the MSE of the measured angle is $\pm 3.1^{\prime \prime}$, and the MSE of the average of the azimuth is $\pm 0.9 "$. The distance is 989.25167 $\mathrm{m}$, and the MSE of the average of the distance is $\pm 0.09 \mathrm{~mm}$. The vertical angle is $3.0231111^{\circ}$, and the MSE of the average of the vertical angle is $\pm 0.7^{\prime \prime}$. The MSE of the position on point 2 is $\pm 6.1 \mathrm{~mm}$.

According to the requirement of the Code for Engineering Survey (2007), the MSE of the rock landslide monitoring points is $\pm 6 \mathrm{~mm}$ so that the MSE of the point position exceeds the limit. If the Paūta criterion is used as the elimination model, then no gross error is found in the monitoring data, which means that the Paùta criterion fails. If the developed model is used as the elimination model, the 10th monitoring datum is found to be the observation containing gross error. After it is removed, the MSE of the point position becomes $\pm 3.6 \mathrm{~mm}$.

The monitoring data of 24 cycles have been processed using the developed model and Paūta criterion. The results show that the average gross error elimination rates of the two methods are $98.9 \%$ and $62.49 \%$, respectively. The main reason why all the gross errors cannot be completely eliminated is that the developed model is limited by the requirements for the differences of several observations containing a residual as large as that of the Paūta criterion[18]. Figure 4 compares the gross error elimination rates of the two methods for 24 cycles of the monitoring data. The model has to be further studied and improved in future research. Nevertheless, the superior practicability of the developed model to that of the Paūta criterion has been tested in 24 cycles of monitoring data.

Table 3. Values of azimuth and residual of point

\begin{tabular}{ccccccc}
\hline Number of observation set & $\mathbf{1}$ & $\mathbf{2}$ & $\mathbf{3}$ & $\mathbf{4}$ & $\mathbf{5}$ \\
\hline Azimuth (degree) & 171.8702736 & 171.8711482 & 171.8705413 & 171.8714427 & 171.8707636 & 171.8708821 \\
\hline Correction (second) & -3.7 & -0.5 & -2.7 & 0.5 & -1.9 \\
\hline Number of observation set & $\mathbf{7}$ & $\mathbf{8}$ & $\mathbf{9}$ & $\mathbf{1 0}$ & $\mathbf{1 1}$ \\
\hline Azimuth (degree) & 171.8711646 & 171.8715540 & 171.8715199 & 171.8736357 & 171.8710978 & 171.8719406 \\
\hline Correction (second) & -0.5 & 0.2 & 0.1 & 8.4 & -0.7 \\
\hline
\end{tabular}

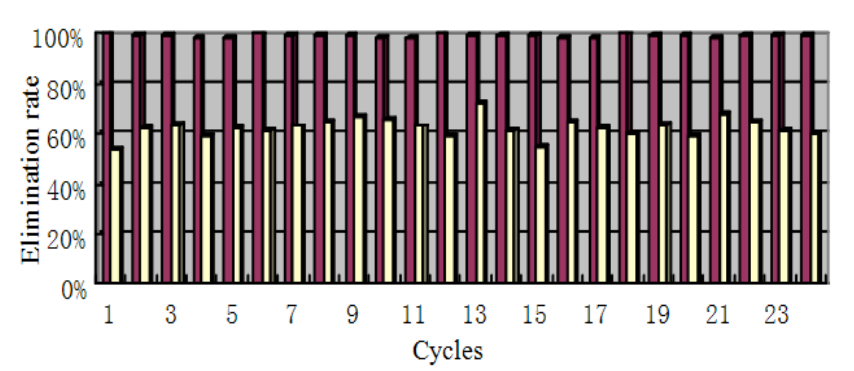

$\square$ the developed model

$\square$ the Paūta criterion

Fig. 4 Comparison of gross error elimination rates of 24 cycles of monitoring data of two methods 


\section{Conclusions}

A total of 24 cycles of the monitoring data on the open-pit iron mines in Qidashan, Yanqianshan, and Dagushan were analyzed. The accuracy requirements of the Code for Engineering Survey (2007) were set as the goal of the slope monitoring of the open pit. The actual probability of the monitoring data containing gross error was calculated through the use of mathematical statistics. An elimination model of the gross error was also developed based on through the use of mathematical statistics. An elimination model of the gross error was also developed based on probability theory. The practicability of the model was tested using the actual monitoring data. The main conclusions are as follows:

1)The probability of the gross errors of the monitoring data obtained by the surveying robot is significantly higher than $0.3 \%$ because of the stability of the measuring equipment and complexity of the changing survey environment. For this reason, the Pauta criterion is not a suitable model of gross error elimination.

2)The elimination model of the gross error is developed based on the actual probability of gross errors of the monitoring data collected by the surveying robot. The practicability of the model is tested against actual monitoring data.
3)This study proves the feasibility of the modeling method with the actual monitoring data as the data source and the accuracy requirements of the monitoring data as the goal. This method can overcome the limitations of modeling methods based on the assumed probability of gross errors and lay the foundation for the rapid processing of monitoring data and early real-time warnings.

However, the elimination rate of gross error of the developed model cannot reach $100 \%$ because the model is limited by the requirements of the differences of several observations containing a residual as large as that of the Paūta criterion. Therefore, this model should be further improved in the future.

\section{Acknowledgements}

The authors are grateful for the support provided by the National Natural Science Foundation of China (No. 41371437), Projects of International Cooperation and Exchanges NSFC (Nos. 51250110531 and 51350110534), Major State Basic Research Development Program (No. 2013CB227902), State Key Laboratory of Geohazard Prevention and Geoenvironment Protection (No. SKLGP2012K009), and "985 Project" of Northeastern University, China.

\section{References}

1. Liu D.-A., Yang Z.-F., Tang C.-H., Wang J. and Liu Y., "An automatic monitoring system for the shiplock slop of Wuqiangxi Hydropower Station”. Engineering Geology, 76 (1-2), 2004, pp. 79-91.

2. Zhao Y.-Q., "A New Type of Automatic Monitoring System of Static and Dynamic Displacement on Dam and Slop". Procedia Engineering. 43, 2012, pp. 387-392.

3. Peng M., Li X.-Y., Li D.-Q., Jiang S.-H., Zhang L.-M., "Slop safety evaluation by integrating multi-source monitoring information". Structural Safety, 49(S1), 2013. pp. 65-74.

4. Xu N.-W., Tang C.-A., Li L.-C., Zhou Z., Sha C., Liang Z.-Z., Yang J.-Y., "Microseismic monitoring and stability analysis of the left bank in Jinping first stage hydropower station in southwestern China". International Journal of Rock Mechanics and Mining Sciences, 48(6), 2011, pp. 950-963.

5. Han X., He M.-C., Zhang B., "Sensitivity analysis for parameters of a monitoring system for steep slopes of open-pit mines". Mining Science and Technology, 19(4), 2009, pp. 441-445.

6. Wang J.-P., Gao J.-X., Liu C., and Wang J., "High precision slope deformation monitoring model based on the GPS/Pseudolites technology in open-pit mine". Mining Science and Technology, 20(1), 2010, pp. 126-132.

7. Tamura Y., Matsui M., Pagnini L.-C., Ishibashi R., Yoshida A., "Measurement of wind-induced response of buildings using RTK-GPS". Journal of Wind Engineering and Industrial Aerodynamics, 90(12-15), 2002, pp. 1783-1793.

8. Gruber V., Marcelino R., Silva J.-B, "Technology PLC-Power line communication used in monitoring systems online". International Journal of Online Engineering, 9(S1), 2013, pp. 22-25.

9. Jiang C.-G., Pang X.-Z., Dong Q.-J., et al., "Development and application of the automatic slope monitoring system in open-pit mine". Beijing Surveying and Mapping, 17(4), 2006, pp. 123-128.
10. Mei W.-S., Zhang Z.-L., Huang Q.-Y., "Application of robotic total station in deformation monitoring". Dam and Safety, 2002 (5), pp. 3335.

11. Mei W.-S., Zhang Z.-L., Guo B.-M., et al., "Study on system of deformation monitoring system with robotic total station". Journal of Wuhan University, 27(2), 2002, pp. 165-171.

12. Mao Y.-H., Wang E.-D., Chen Y.-S., Yang Y.-Z., "Problems and Solutions to Developing Automatic Deformation Monitoring Software for Surveying Robot with GEOCOM ". Metal Mine, 2010 (3), pp.94-96. 13. Ma Z.-L., Ji C.-D., and Ren D.-F., "Application of GPS and the surveying robot joint operation mode in open-pit mine deformation monitoring". Mine Surveying, 2007 (1), pp.41-43.

14. Chang M., Yuan H., "Paūta criterion and the abnormal value elimination". Journal of Zhengzhou University of technology, 18(1), 1997, pp.84-88.

15. Wang C.-C., Ma M.-J., Ma N., Zhang W.-D., Shen R.X., "Algorithm for land area measurement with GPS technology". Journal of Shandong University of Technology, 27(4), 2013, pp.64-68.

16. Zhai W.-K., Xu B.-J., Fang J.-CH., "Research on the method of estimating the uncertainty of Beidou satellite positioning system". Measurement \& Control Technology, 2005 (3), pp.32-34.

17. Yao X.L., Chen H.-L., Zhao X., Guo S.-J., "Weak link determination of anti-shock performance of shipboard equipment based on Paūta criterion ". Chinese Journal of Ship Research, 2(5), 2007, pp.10-14.

18. Mao Y.-C., Wang E.-D., Xiu C.-H., "A new method data caper method for rejecting the deformation monitoring data with the gross errors ".Journal of Northeastern University, 2011(7), pp.1020-1023. 